\title{
Evaluating innovation. Part 1: The concept of progressive scholarly acceptance
}

\author{
Zane Schnurman, BA, and Douglas Kondziolka, MD \\ Department of Neurosurgery, NYU Langone Medical Center, New York, New York
}

\begin{abstract}
Understanding how the relevant medical community accepts new therapies is vital to patients, physicians, and society. Increasingly, focus is placed on how medical innovations are evaluated. But recognizing when a treatment has become accepted practice-essentially, acceptance by the scientific community-remains a challenge and a barrier to investigating treatment development. This report aims to demonstrate the theory, method, and limitations of a model for measuring a new metric that the authors term "progressive scholarly acceptance."
\end{abstract}

A model was developed to identify when the scientific community has accepted an innovation, by observing when researchers have moved beyond the initial study of efficacy.

This model could enable further investigations into the methods and influences of treatment development.

http://thejns.org/doi/abs/10.3171/2015.1.JNS142661

KEY WORDS science; innovation; standard of care; surgery; idea

$\mathrm{I}$ N 1993, the US Supreme Court ruled that an essential component for a treatment to be considered standard practice is acceptance from the relevant scientific community. ${ }^{4}$ Yet, how the medical community comes to embrace new treatment options is not always clear.

The largest determinant of whether physicians agree on a treatment's efficacy is likely the results of scientific studies. ${ }^{5}$ However, questions remain as to which kinds of studies and what volume of investigations are required to move a treatment from experimental to validated. Additionally, there may be numerous external forces that contribute to an innovation's pace and process. ${ }^{8}$ Pressures from the lay community, regulatory rulings, and funding sources are examples of factors that could play a role in the evolution of a new idea. All are components worthy of study. Considering the wide implications of which treatments become designated as acceptable, further investigation of how a therapy moves from conception to acceptance is warranted.

This is particularly important in fields in which treatment development is less standardized. For many new drugs, the developmental pathway in the United States is laid out from preclinical studies through Phase 3 randomized controlled trials by FDA regulations. Other countries use their own processes. But for other treatments, particularly surgeries, ${ }^{2}$ the progression of a procedure to community acceptance is less clear.
A significant challenge for investigating treatment development is the selection of an applicable end point that represents community acceptance for procedures that did not develop along a standard drug-based path. This report aims to demonstrate the theory, method, uses, and limitations of a new model for a metric that we call progressive scholarly acceptance (PSA). The model aims to identify how and when the scientific community has accepted an innovation by observing when scholars have moved beyond the initial study of the idea to studies that refine the idea. This paper will mostly focus on PSA of surgical procedures, but the theory may be applicable to a variety of medical and scientific fields.

\section{Theory}

Progressive scholarly acceptance is a new metric that attempts to identify when an idea or medical innovation moves from the experimental fringe to the academic mainstream. It is based on a view of surgical innovation as continually building on past innovations.

Riskin et al. explored historical patterns of surgical innovation and, through the lens of the plentiful research on business innovation, created new nomenclature to categorize contributions based on clinical impact. ${ }^{9}$ They argued for two phases of surgical innovation: an "expand-

ABBREVIATIONS PSA = progressive scholarly acceptance; $\mathrm{TN}=$ trigeminal neuralgia .

SUBMITTED November 20, 2014. ACCEPTED January 13, 2015.

INCLUDE WHEN CITING Published online August 7, 2015; DOI: 10.3171/2015.1.JNS142661.

DISCLOSURE The authors report no conflict of interest concerning the materials or methods used in this study or the findings specified in this paper. 
ing period" and a "refining period." An expanding period occurs when a significant innovation spurs rapid change that considerably alters patient care. A refining period is a time when new developments merely improve the existing method with relatively modest clinical impact. These refinements increase efficiency, decrease costs, or moderately improve outcomes, but do not have a huge bearing on patient care. The authors described surgical history as having an "ebb-and-flow" pattern, where an expanding technology leads to rapid clinical change followed by a period of refinement when those changes are moderately improved. ${ }^{9}$

In their work, Riskin et al. use this theory to describe development of whole fields over centuries, but the concepts of expanding and refining innovations can also be applied to a narrower perspective. ${ }^{9}$ In fact, depending on the focus, a single innovation could be considered an expanding or refining technology. One example is stereotactic radiosurgery in neurosurgery. When viewed in a broader context, the invention of radiosurgery is the enabling technology and new applications that arose over the subsequent decades, such as its use for trigeminal neuralgia (TN) or brain metastases, were new refinements. But from a narrower perspective, the use of radiosurgery for TN could be considered an expanding innovation, as it had significant impact on certain patients' care and enabled considerable research into even further refinement. Examples are its application in certain TN subpopulations, such as patients with multiple sclerosis, and in technical refinements such as radiation dose.

In this view, broader innovations give rise to refining innovations that themselves result in more narrow refinements. An appropriate visual representation of this repeating pattern is a diagram of a branching tree, where each branch is a new innovation (Fig. 1). Any branch could be selected and viewed as the broad innovation, and the innovations branching from it viewed as the refinements.

Key to the PSA theory is the recognition that prior to refinement comes acceptance of the broad, initial innovation. That is, before an investigator begins to study a refinement, he or she believes in the procedure they are building upon. Additionally, when a report is published, it reveals the views of more than just its authors, as the journal reviewers must acknowledge the appropriateness of the idea for study. As studies diffuse, more investigators are likely to accept the procedure and begin their own refinements, further publishing and perpetuating the procedure's usefulness. At some point, assuming continued procedural success, the volume of reports focused on refinements will eclipse the number of studies testing the initial question of efficacy. This is an indication of community acceptance, and what we term PSA. Progressing-or moving beyond the initial question-is a signal of acceptance from the relevant community.

It is crucial to point out that community acceptance of a certain procedure does not mean it is objectively efficacious; it only means that the community at large believes in its efficacy at some level. There are many examples, such as autologous bone marrow transplantation for breast cancer, ${ }^{11}$ of a treatment initially being accepted by the scientific community and considered to be an optimal ther-

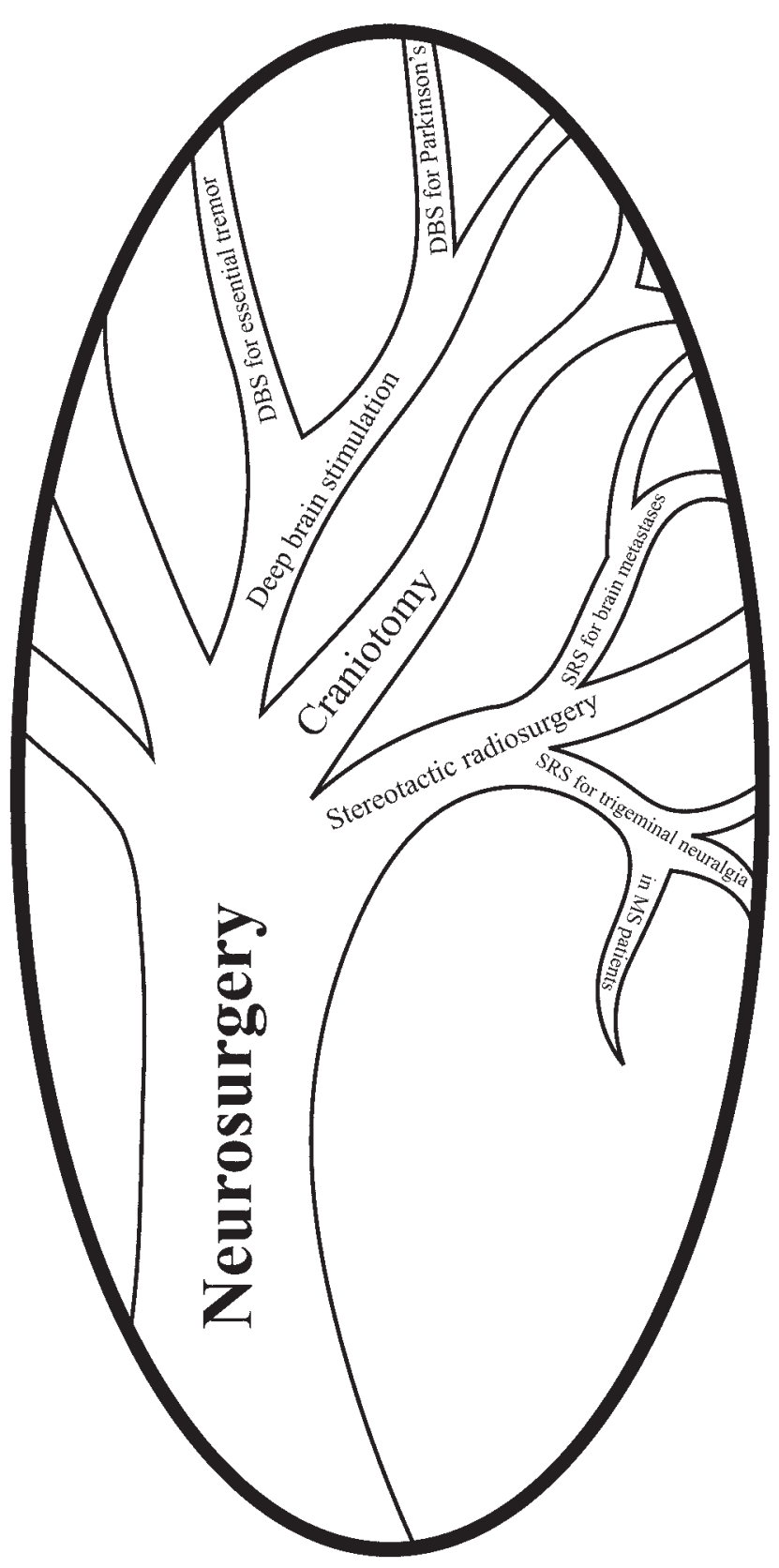

FIG. 1. Scientific innovation and refinement as a branching tree. DBS = deep brain stimulation; MS = multiple sclerosis; SRS = stereotactic radiosurgery.

apy, and then additional studies demonstrated its ineffectiveness.

The goal of the PSA model is not to assess effectiveness but to use quantifiable, literature-based documentation to determine when the community accepts a procedure. Second, it can be used to study how the community accepts a procedure. This has significant value for studying development. PSA can be used as an end point for acceptance, and then the scientific characteristics of studies, the norms of a specific community, potential societal factors, and other variables can be described to identify how each influences innovation and movement toward acceptance. 


\section{Methods}

The PSA model uses the scientific literature to assess how a topic is proposed, evaluated, disseminated, and adopted. The model is a bibliometric analysis (i.e., a measurement of texts and information ${ }^{3}$ ) but is distinct from other well-known bibliometric tools such as impact factor and the h-index, which focus on the citation impact of the article or authors. The PSA model assesses the progression of a topic over time, similar to a literature review, but instead of selecting key articles, all literature found in selected databases is included in the analyses.

It may seem counterintuitive for a model that assesses the spread of acceptance to not differentiate article quality, as higher-quality articles in more renowned journals are likely to be more widely read and the findings perhaps accepted by more physicians. But instead of weighting articles with a metric such as journal impact factor, the influence of an article (as well as ancillary forces that are harder to measure, such as conference meetings/proceedings, personal correspondences, and related writings) is predicted to present itself when it instigates more investigations on the topic by more investigators. These subsequent publications are included in the PSA model. This will allow for observing the topic's reception: Procedures met with skepticism should inspire further efforts to validate findings, while articles whose findings are endorsed will lead to more refining studies.

This is one of the keys to assessing PSA. Instead of creating a web of impact through citation, the model takes annual cross sections of topic publications to monitor the academic exchange and how it progresses. High-profile reports, regulatory agency rulings, targeted advertising, or many other factors could impact the adoption process, and the PSA model aims to capture the result to enable evaluation.

The first step in modeling PSA is to select which procedures will be evaluated. This will depend on the research question. If examining developmental patterns in a particular field (as we do for neurosurgery in Part 2 of this report $\left.{ }^{10}\right)$, significant innovations that span the relevant field may be selected and their results reviewed. If studying how a particular report characteristic impacts acceptance, such as the influence of funding sources, data on two groups of procedures with different funding sources could be collected and compared.

Once selected, a procedure should be searched in appropriate databases (with all essential keywords, including historical names, if applicable), and all research publications should be reviewed in chronological order, starting with the introductory report. Only original research reports, not reviews or editorials, should be included. Additional exclusions, such as preclinical animal studies, may be applicable, depending on the aim of the study. Additional characteristics recorded during the literature review will again depend on the research question, though crucial for the PSA model is year published and whether each report aims to answer an initial question (usually broad efficacy), or is a refinement. A refinement could be an investigation of the procedure on a modified method, a more narrow or distinct target population, or many other variations.
Here are examples from modeling PSA of radiosurgery for TN. A 1996 report assessed general efficacy in 50 separate TN patients with its main goal being to "reevaluate the early anecdotal success of TN radiosurgery." " This would be considered an initial investigation. Whereas, a 2001 article by some of the same authors sought to investigate if increasing the irradiated nerve length improved pain outcomes. ${ }^{6}$ This would be a refining investigation.

The language in the introduction of a report sets the tone and can often indicate whether that work is an initial investigation of the idea or a refining study. In initial investigations, the author generally suggests that the community remains unconvinced of the procedure prior to their investigation-usually citing early work, or lack thereof, and commenting on the need for further study of efficacy. In refining investigations, authors tend to introduce the topic as generally accepted and cite the initial investigations as evidence of efficacy before moving on to the new question they aim to address.

After data collection, there are two main PSA analyses: annual and compounding. We will demonstrate these analyses with results from our model of radiosurgery for $\mathrm{TN}$. In the annual analysis, the number of initial and refining publications per year are plotted on a line graph (Fig. 2 upper). This analysis allows for more time-sensitive review of publication patterns, which is best used to assess changes following specific incidents (e.g., a regulatory ruling). But the annual changes tend to be more fickle, changing rapidly from chance and varied influences, and the point when the annual number of refining publications surpasses the annual number of initial studies may not be the best measure of acceptance. A more conservative measure for acceptance is found using the compounding analysis. In the compounding analysis, the total number of initial and refining publications is compounded each year as time passes (Fig. 2 lower). The year when the compounded number of refining articles surpasses the compounded number of initial articles is the best measure of PSA. This is the point when the total volume of articles written on refining innovations surpasses the volume of articles assessing basic efficacy. Reaching this point requires sustained refinement, with the rate of publication of refining articles remaining higher than that of initial efficacy reports. This means that the majority of research has moved on to the refining questions, implying the scientific community has accepted the answer to the initial question of efficacy.

For TN radiosurgery (Fig. 2), it can be seen that the annual study of refinement grew considerably and surpassed the study of initial efficacy in 2000 . From then on, refinement investigations were sustained at higher levels, and by 2002 , the total number of articles written about refinements for TN radiosurgery had eclipsed the number of articles assessing efficacy, as seen in the compounding plot (Fig. 2 lower). This is the point of PSA, and that year is the end point for community acceptance in the PSA model.

For $\mathrm{TN}$ radiosurgery, the community belief in efficacy aligns with the objective assessment of efficacy. The National Institute of Clinical Excellence in the United Kingdom released a systematic review endorsing radiosurgery for TN in 2004, with all of the evidence for Gamma knife 

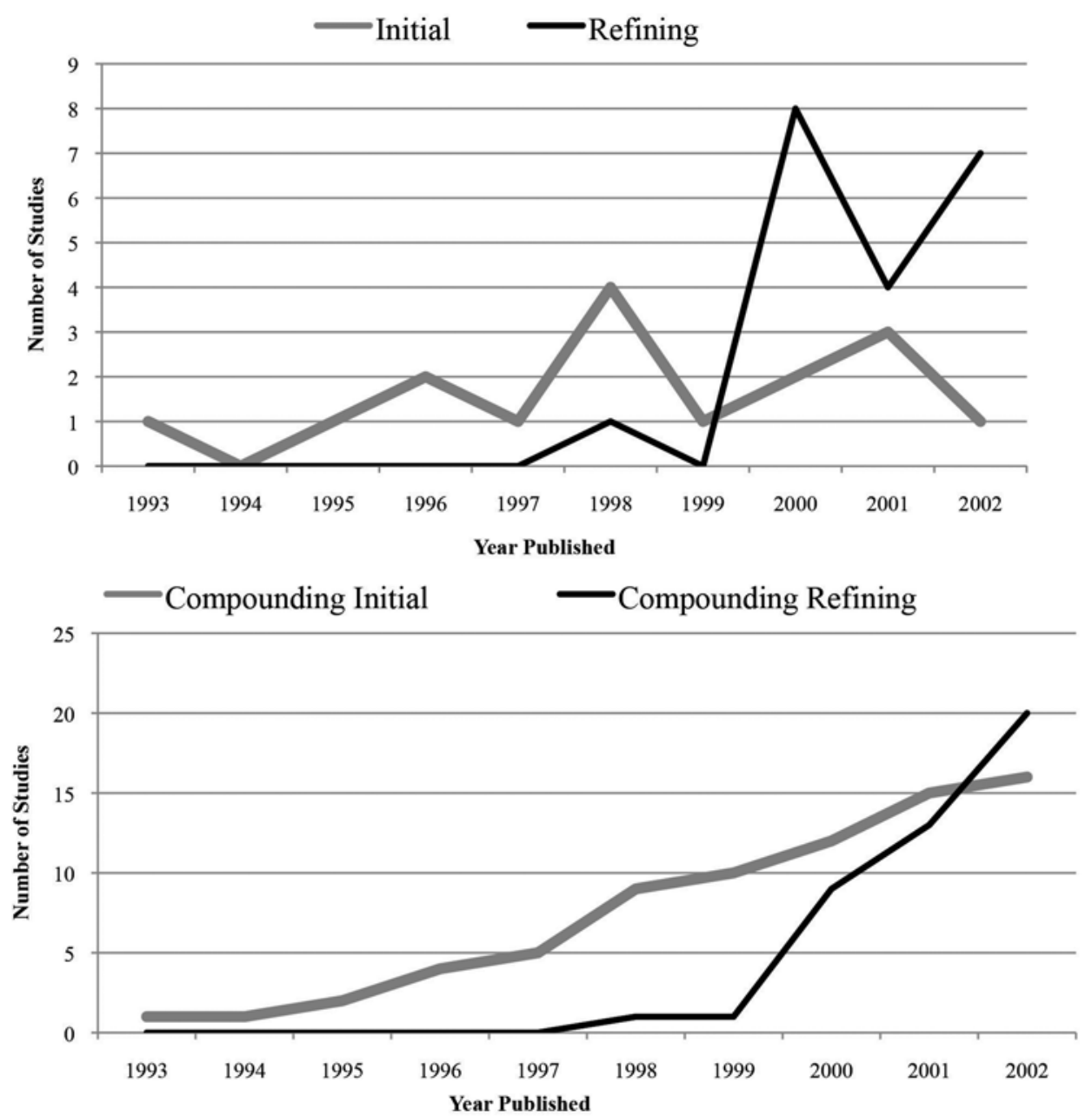

FIG. 2. Upper: The number of initial and refining publications per year are plotted on a line graph (e.g., radiosurgery for TN). Lower: The compounded number of initial and refining publications per year are plotted on a line graph (e.g., radiosurgery for TN).

radiosurgery published in 2002 or earlier. But another major value of PSA will be in investigating when acceptance does not agree with objective reviews of efficacy and which factors outside of scientific evidence change the community's belief in efficacy.

\section{Limitations}

There are several limitations to the PSA model. The first is the time-intensive nature of producing the model. Currently, each report must be manually assessed to characterize the investigation as initial or refining. With wider utilization, this limitation could be overcome if authors were to declare which areas they were building on, similar to entering a keyword. This would allow for automated analysis.

Another problem is the delay from author acceptance until publication. Gathering data and completing a study takes time, and the PSA model does not register acceptance of the author until those studies are published. This is a real delay between the work performed and the publication.

Another timing issue is how well acceptance from aca- demic medicine corresponds to clinician utilization. Clinicians that participate in clinical research are more likely, by profession, to be early adopters, ${ }^{1}$ and may accept and begin refining an innovation before noninvestigative clinicians accept it. The PSA model is clearly an assessment of scholarly views, and the correspondence between PSA and clinical use requires further investigation.

The model also fails to take into account published reviews and editorials. This is because they are not original studies, and cannot be readily classified as initial efficacy or refining investigations. Additionally, the timing of release of these types of articles does not necessarily correspond with the most up-to-date science. For example, a review may detail the results from older efficacy studies to remind readers about effectiveness long after investigators have moved onto refinement topics. But still, these methods of publication are part of the scientific conversation and surely have a role in demonstrating community perspective, so their absence is relevant.

\section{Conclusions}

The PSA metric fills a vital role as an end point for 
community acceptance of science. Despite its limitations, the PSA model we propose has considerable use in enabling investigators to further study how clinical medicine (or other science) evolves in different circumstances, and how varying factors influence societal acceptance of that science. These types of investigations will be critical to understanding treatment development and could inform strategies aiming to reform or regulate medical innovation.

\section{References}

1. Berwick DM: Disseminating innovations in health care. JAMA 289:1969-1975, 2003

2. Biffl WL, Spain DA, Reitsma AM, Minter RM, Upperman J, Wilson M, et al: Responsible development and application of surgical innovations: a position statement of the Society of University Surgeons. J Am Coll Surg 206:1204-1209, 2008

3. Daim TU, Rueda G, Martin H, Gerdsri P: Forecasting emerging technologies: Use of bibliometrics and patent analysis. Technol Forecast Soc 73:981-1012, 2006

4. Daubert v Merrell Dow Pharmaceuticals, 509 US 579, 589 (1993)

5. Ellis J, Mulligan I, Rowe J, Sackett DL: Inpatient general medicine is evidence based. Lancet 346:407-410, 1995

6. Flickinger JC, Pollock BE, Kondziolka D, Phuong LK, Foote RL, Stafford SL, et al: Does increased nerve length within the treatment volume improve trigeminal neuralgia radiosurgery? A prospective double-blind, randomized study. Int J Radiat Oncol Biol Phys 51:449-454, 2001

7. Kondziolka D, Lunsford LD, Flickinger JC, Young RF, Vermeulen S, Duma CM, et al: Stereotactic radiosurgery for trigeminal neuralgia: a multiinstitutional study using the gamma unit. J Neurosurg 84:940-945, 1996
8. Rogers EM: Diffusion of Innovations, ed 5. New York: Free Press, 2003

9. Riskin DJ, Longaker MT, Gertner M, Krummel TM: Innovation in surgery: a historical perspective. Ann Surg 244:686693, 2006

10. Schnurman Z, Kondziolka D: Evaluating innovation. Part 2: Development in neurosurgery. J Neurosurg [epub ahead of print August 7, 2015. DOI: 10.3171/2015.1.JNS142664]

11. Welch HG, Mogielnicki J: Presumed benefit: lessons from the American experience with marrow transplantation for breast cancer. BMJ 324:1088-1092, 2002

\section{Author contributions}

Conception and design: both authors. Acquisition of data: Schnurman. Analysis and interpretation of data: both authors. Drafting the article: both authors. Critically revising the article: both authors. Reviewed submitted version of manuscript: both authors. Approved the final version of the manuscript on behalf of both authors: Kondziolka. Statistical analysis: Schnurman. Administrative/technical/material support: Kondziolka. Study supervision: Kondziolka.

\section{Supplemental Information \\ Companion Paper}

Schnurman Z, Kondziolka D: Evaluating innovation. Part 2: Development in neurosurgery. DOI: 10.3171/2015.1.JNS142664.

\section{Correspondence}

Douglas Kondziolka, Department of Neurosurgery, NYU Langone Medical Center, 530 First Ave., Ste. 8R, New York, NY 10016. email: douglas.kondziolka@nyumc.org. 